\title{
MRI amygdala volume in Williams Syndrome
}

\author{
Liliana Capitão $^{\mathrm{a}, 1}$, Adriana Sampaio ${ }^{\mathrm{a}, 1, *}$, Cassandra Sampaio ${ }^{\mathrm{a}}$, Cristiana Vasconcelos ${ }^{\mathrm{b}}$, \\ Montse Férnandez ${ }^{c}$, Elena Garayzábal ${ }^{\text {d }}$, Martha E. Shenton ${ }^{\text {e,f }}$, Óscar F. Gonçalves ${ }^{a}$ \\ ${ }^{a}$ Neuropsychophysiology Lab, CIPsi, School of Psychology, University of Minho, 4710-057 Braga, Portugal \\ ${ }^{\mathrm{b}}$ Department of Neuroradiology, Hospital Geral Santo António, Porto, Portugal \\ ${ }^{\mathrm{C}}$ Genetic Molecular Unit, Galician Public Foundation of Genomic Medicine, University of Santiago de Compostela, Spain \\ ${ }^{\mathrm{d}}$ Department of Linguistics, University Autónoma de Madrid, Madrid, Spain \\ e Psychiatry Neuroimaging Laboratory, Department of Psychiatry, Brigham and Women's Hospital, Harvard Medical School, Boston, MA, USA \\ ${ }^{\mathrm{f}}$ Clinical Neuroscience Division, Laboratory of Neuroscience, Department of Psychiatry, VA Boston Healthcare System, and Harvard Medical School, Brockton, MA, USA
}

\section{A R T I C L E I N F O}

\section{Article history:}

Received 24 May 2011

Accepted 27 May 2011

Available online 12 July 2011

\section{Keywords:}

Williams Syndrome

Hypersociability

Amygdala

Neurodevelopment

\begin{abstract}
A B S T R A C T
One of the most intriguing characteristics of Williams Syndrome individuals is their hypersociability. The amygdala has been consistently implicated in the etiology of this social profile, particularly given its role in emotional and social behavior. This study examined amygdala volume and symmetry in WS individuals and in age and sex matched controls. Magnetic resonance imaging scans were obtained on a GE 1.5-T magnet with 1.5$\mathrm{mm}$ contiguous slices and were used to measure whole gray matter, white matter and cerebrospinal fluid volumes, as well as amygdala volume (right and left). Results revealed significantly reduced intracranial volume in individuals with WS, compared with controls. There were no differences between groups in absolute amygdalae volume, although there was a relative increase in amygdalae volumes, when adjusted for total intracranial content. There were no inter-hemispheric differences in amygdalae volumes in both groups. These results suggest a relative increase in amygdala volume in WS compared with healthy controls that likely reflects abnormal neurodevelopmental processes of midline brain structures.
\end{abstract}

(c) 2011 Elsevier Ltd. All rights reserved.

\section{Introduction}

Williams Syndrome (henceforth WS) is a rare genetically determined neurodevelopmental disorder with an estimated incidence of 1 in 7500 to 20 in 30,000 births (Strømme, Bjømstad, \& Ramstad, 2002) and is caused by a hemideletion on chromosome 7q11.23 (Ewart et al., 1993; Peoples et al., 2000). Individuals with WS display an unusual phenotype, which includes a distinctive profile of physical, medical, neurocognitive, behavioral, and neuroanatomical characteristics. One of the most striking features of individuals with WS is a distinctive social-affective profile, characterized by high sociability, disinhibition, over-friendliness (Bellugi, Adolphs, Cassady, \& Chiles, 1999; Klein-Tasman \& Mervis, 2003), and strong empathy (Klein-Tasman \& Mervis, 2003). Indeed, individuals with this syndrome reveal a facility for making social contacts as well as being particularly sensitive to others' feelings. Many aspects of this social profile (in particular the attraction to social interaction) are evident in early childhood, suggesting that this aspect of the WS phenotype may be independent of other cognitive impairments and pervasive across age spans (Jones et al., 2000).

\footnotetext{
* Corresponding author.

E-mail address: adriana.sampaio@psi.uminho.pt (A. Sampaio).

1 Share equal first authorship.
} 
The etiology of this social phenotype is still unknown. However, the role of the amygdala has consistently been hypothesized as being implicated in the WS social phenotype (Bellugi et al., 1999; Capitao et al., 2011; Meyer-Lindenberg, Hariri, et al., 2005). In general, the amygdala modulates a large variety of psychological functions, such as emotional expression and processing, including emotional facial expression recognition (e.g., Adolphs, Tranel, Damasio, \& Damasio, 1994, 1995), emotional memory (e.g., Cahill, Babinsky, Markowitsch, \& McGaugh, 1995), emotional auditory recognition (e.g., Scott et al., 1997) and fear conditioning (e.g., LeDoux, 1998). It is thus an important component of the neural network that underlies social cognition (Adolphs, 1999, 2003), playing a key role in processing social-related stimuli (Adolphs, 1999, 2003) and monitoring environmental events such as danger (Amaral, 2002).

These findings, have led some researchers to propose that abnormal amygdala processing may be responsible for the hypersocial behavior typical of WS phenotype (Galaburda \& Bellugi, 2000; Reiss et al., 2004). In fact, researchers have used neuroimaging technology in an attempt to tackle the neural substrates involved in the WS social-affective profile (MeyerLindenberg, Hariri, et al., 2005). However, structural magnetic neuroimaging studies have produced inconsistent results regarding volume and gray matter density of the amygdala in WS. More specifically, absolute volume of the amygdala in WS was found to be similar to comparison groups, using both manual segmentation methods [either analyzed within limbic system structures (Jernigan, Bellugi, Sowell, Doherty, \& Hesselink, 1993) or individually (Martens, Wilson, Dudgeon, \& Reutens, 2009; Reiss et al., 2004)] and automatic segmentation methods (e.g., voxel-based morphometry) (Chiang et al., 2007; Reiss et al., 2004). In parallel, disproportional enlargement of this structure, characterized by relative increases in volumes and gray matter density have also been reported in WS (Martens et al., 2009; Reiss et al., 2004). Contradictory findings, however, have also been reported by Meyer-Lindenberg and collaborators, where a relative increase of amygdala gray matter density was not observed in WS (Meyer-Lindenberg et al., 2004). Negative findings with respect to increased amygdala volume have also been reported in a post-mortem study (Galaburda \& Bellugi, 2000). Such inconsistencies among studies are likely due to the use of different methods to analyze amygdala structure and volume and the use of different groups of individuals with WS (with intelectual disabilities or normal IQ), making it difficult to take definitive conclusions concerning amygdala volumetric differences in WS.

Given the inconsistent findings for amygdala involvement in WS and our previous neuropsychological study partially supporting the amygdala contribution to WS hypersociability (Capitao et al., 2011), the aim of this study was to analyze amygdala volumes in individuals with WS and in typically developing controls matched for sex and age, by employing a manual tracing method performed in realigned native space. This manual tracing methods allows for a more rigorous analysis, in comparison with surface analysis, since this method uses simultaneous images of the coronal, sagittal and axial views (Martens et al., 2009).

\section{Method}

\subsection{Participants}

Study participants included 17 individuals with WS ( 7 males and 10 females) (19.29 \pm 6.29 ; age-range: 11-34 years) and 16 healthy controls individually matched for sex and age ${ }^{2}$ ( 7 males and 9 females) (19.88 \pm 6.67 ; age-range: 11-34 years). Mean Full Scale IQ was $52.27( \pm 9.79)$ for WS and $108( \pm 10.31)$ for controls. WS diagnoses were made by FISH confirmation of elastin gene deletion (Ewart et al., 1993). Controls were typically developing individuals without evidence of psychiatric, neurological disorder or cognitive impairment. After a complete description of the study, each participant or their guardians gave written informed consent for the participation in the study via consent forms. All participants were right-handed, determined through clinical interview.

Table 1 displays socio-demographic characteristics of the sample. There was no significant group differences in the sociodemographic characteristics, including age $(t(31)=-.257, p=.80)$ and socio-economical status-Graffar index $(Z=-.160$, $p=.90)$, although the groups did differ in education level $(Z=-2.70, p<.01)$ and in $\operatorname{IQ}(Z=-4.33, p<.001)($ data is shown in Table 1).

\subsection{MRI acquisition and processing}

MRI images were obtained on a $1.5 \mathrm{~T}$ General Electric system (GE Medical Systems). The scans acquisition protocol consisted of contiguous 1.5-mm coronal T1 slices of the whole brain and an axial PD/T2 sequence. The parameters used were echo time: $5.0 \mathrm{~ms}$, repetition time: $35 \mathrm{~ms}$, flip angle: $45^{\circ}$, acquisition matrix: $256 \times 192$, voxel dimensions: $0.9375 \mathrm{~mm} \times 0.9374 \mathrm{~mm} \times 1.5 \mathrm{~mm})$. Images were aligned and resampled $\left(0.9375 \mathrm{~mm}^{3}\right.$, cubic interpolation) and an atlas-based expectation maximization segmentation was used (Pohl, Bouix, \& Kikinis, 2004).

\subsection{Region of interest definition}

The amygdala was outlined manually using the 3D Slicer Software (http://www.slicer.org/) in realigned native space coronal images (Fig. 1), with guidance from sagittal and coronal views. Segmentation was performed in coronal slices from anterior to posterior. In the anterior border, the first slice of the amygdala was identified at the level where the white matter

\footnotetext{
${ }^{2}$ One participant was matched for two subjects with WS.
} 
Table 1

Socio-demographic characteristics.

\begin{tabular}{|c|c|c|c|c|}
\hline & \multicolumn{2}{|l|}{ WS $(N=17)$} & \multicolumn{2}{|c|}{ Control group $(N=17)$} \\
\hline & $M(\mathrm{SD})$ & Range & $M(\mathrm{SD})$ & Range \\
\hline Age & $19.29(6.3)$ & $11-34$ & $19.88(6.7)$ & $11-34$ \\
\hline \multirow[t]{3}{*}{ Full Scale IQ } & $52.27(9.8)$ & $40-62$ & $108(10.3)$ & $90-124$ \\
\hline & & WS $(N=17)$ & \multicolumn{2}{|c|}{ Control group $(N=17)$} \\
\hline & Mdn & Range & Mdn & Range \\
\hline Level of education & 9 & $6-9$ & 11.5 & $6-15$ \\
\hline Socio-economical status & 3.5 & $1-5$ & 3.25 & $1-4$ \\
\hline \multicolumn{5}{|l|}{ Sex } \\
\hline Male & 7 & $41.18 \%$ & 7 & $41.18 \%$ \\
\hline Female & 10 & $58.82 \%$ & 10 & $58.82 \%$ \\
\hline
\end{tabular}

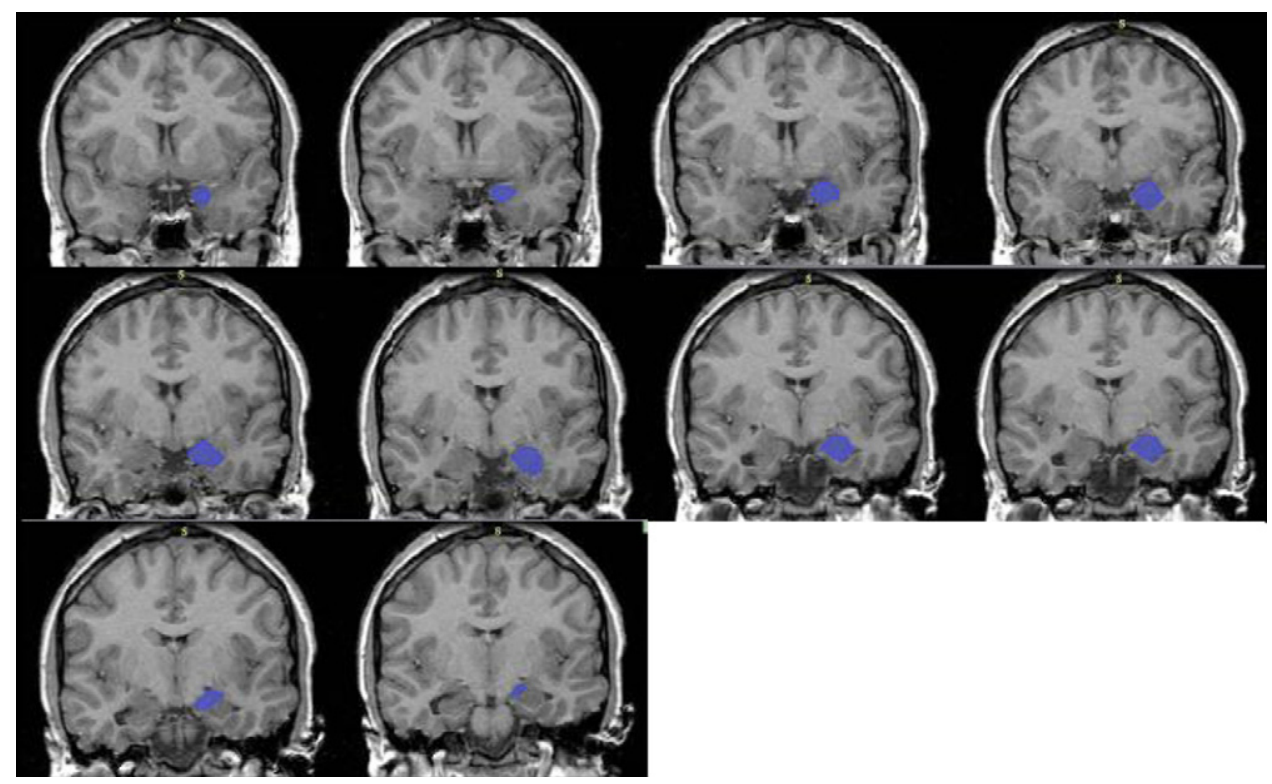

Fig. 1. Manually traced amygdala from anterior to posterior border (left hemisphere).

of the anterior commissure first crossed the midline (in axial plane). The inferior, medial and lateral borders were determined by the boundary between amygdala and adjacent white matter (von Gunten, Fox, Cipolotti, \& Ron, 2000). We considered the superior border of the amygdala as the line forming the entorhinal sulcus extending to the temporal stem. The posterior boundary of amygdala was formed by the disarticulation with the hippocampus (von Gunten et al., 2000). The asymmetry index for amygdala was computed according to the following expression: $(L-R) / 0.5(L+R)$, where $L$ and $R$ refer to absolute volumes in the left and right hemispheres (Galaburda, Rosen, \& Sherman, 1990). Positive values indicate that the amygdala was larger in the left hemisphere.

Adjusted amygdala was computed as the ratio between absolute volume and total intracranial content (TIC), which was computed as the sum of gray matter, white matter and cerebrospinal fluid (CSF) volumes.

\subsection{Reliability analysis}

Two raters, blind to study hypothesis, group membership, and to subject age and sex, measured both left and right amygdala for all subjects with an intra-class reliability of .968 and .963 , respectively.

\subsection{Data analysis}

Data were computed using the SPSS software for Windows 16.0 version. For the analyses, it was calculated the mean values of the two raters for right and left amygdala. The criteria of normality (Kolmogorov-Smirnov and Shapiro-Wilk tests) and variance homogeneity (Levene and Box tests) were evaluated. A mixed analysis of variance was used to determine 


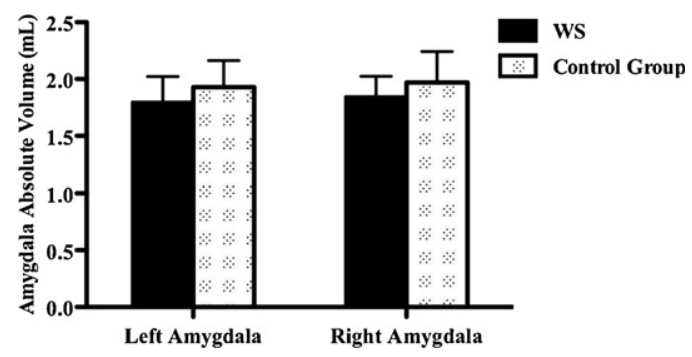

Fig. 2. Amygdala absolute volumes (left and right) in WS and control groups.

amygdala volume differences between the WS and typically developing individuals (controls). Thus group (WS and controls) was used as the between-subject factor and hemispheric side (left vs. right) as the within factor. If a main effect for group was found, then a Student $t$ test was used to test the mean difference between groups. Effect sizes were also calculated for group differences. A $p$ value less than .05 was assumed to denote a significant difference.

\section{Results}

We compared overall brain volumes between the two groups and observed that WS subjects showed a significant reduction in gray matter $(t(30)=2.55, p<.05$; effect size $=.90)$, white matter $(t(30)=4.43, p<.001$; effect size $=1.57)$, and CSF volumes $(t(30)=4.17, p<.001$; effect size $=1.48)$. As a consequence, TIC was significantly reduced in the clinical group $(t(30)=4.65, p<.001$; effect size $=1.72)$.

Repeated-measures analysis of variance of amygdala absolute volumes did not reveal a side effect (left vs. right) $[F(1$, $31)=3.58, p=.07]$, group effect $[F(1,31)=2.83, p=1.02]$ nor an interaction between side and group $[F(1,31)=.003, p=.86]$ (Fig. 2). When amygdala volumes ${ }^{3}$ were adjusted and then analyzed (adjusted to TIC), we observed a group effect $[F(1$, $30)=7.74, p<.01]$, but no side $[F(1,30)=3.99, p=.05]$ or side by group interaction effects $[F(1,30)=.01, p=.82]$ (Fig. 2B). Specifically, individuals with WS evinced a relative increase in left and right amygdala volumes (11.60\% relative increase in left amygdala, $M=1.54, \mathrm{SD}=.19 ; 11.27 \%$ relative increase in right amygdala, $M=1.58, \mathrm{SD}=.17$ ), compared with typically developing individuals $(M=1.38, \mathrm{SD}=.17, t(30)=2.57 ; p<.05$; effect size $=0.41 ; M=1.42, \mathrm{SD}=.17, t(30)=2.71 ; p<.05$; effect size $=0.36$, respectively) .

\section{Discussion}

The present study confirms an overall reduction in brain volumes in the WS group, including a reduction in overall gray matter, white matter, and CSF, compared with controls. Further, while absolute volume of the amygdala were similar in both groups, when it was adjusted to total intracranial contents, there was a relative increase in amygdala volume in WS subjects compared with controls. These findings are in accordance with other reports (Chiang et al., 2007; Jernigan et al., 1993; Martens et al., 2009; Reiss et al., 2004), but in contrast to findings that did not show gray matter increases in amygdala volume in WS (Meyer-Lindenberg et al., 2004), as well as in contrast to findings that have reported reduced amygdala volume in a post-mortem study by Galaburda and Bellugi (2000).

Different factors may contribute to these contradictory findings: (a) different methods were used in beuroimaging vs. postmortem studies; (b) different participant diagnostic criteria were used (e.g., genetic confirmation using FISH vs. behavioral phenotype and neuroendocrine markers); (c) different types of image acquisition were used (e.g., slice thickness 5-mm brain slices with 2.5-mm gaps in-between slices vs. image techniques that permit 1-2-mm resolution); (d) different segmentation used in native vs. standard space; (e) different post-processing analysis was used (e.g., automatic and semiautomatic volumetric analysis vs tensor/voxel-based morphometry), and, finally; (f) there were different sample sizes across studies, and different characteristics of the comparison groups (e.g., group of individuals with WS with normal IQ vs. with intellectual disabilities and controls groups-Down Syndrome vs healthy controls).

Our study employed a manual segmentation method on realigned and re-sampled scans, with defined landmarks and reliability scores $>.95$. This manual method has an advantage over automatic segmentation processing in that we are analyzing a neurodevelopmental disorder characterized by cerebral hypoplasia, with different patterns of brain tissue development, whereby normalizing the brains of individuals with WS to brain atlases like Talairach or MNI neglects the specificity of WS brain architecture. Moreover, the use of these atlases in spatial normalization and co-registering is generally limited to individuals exhibiting similar phenotypes whereas different and specific brain atlases are recommended for use with other populations with different genetic and environmental factors (Altaye, Holland, Wilke, \& Gaser, 2008; Tang

\footnotetext{
${ }^{3}$ For these analyses, only 15 subjects from the control group were considered, since total intracranial and gray matter volumes from one participant were not available (due to technical complications).
} 
et al., 2010), particularly because display atypical brain morphometric patterns (e.g., for WS, see Sampaio et al., 2010; Thompson et al., 2005).

Finally, it is worth remarking that findings of disproportionate increases in amygdala volumes, as reported in the present study, suggests that abnormal functioning of this structure may underlie the enhanced sociability observed in WS (MeyerLindenberg, Hariri, et al., 2005). Indeed, this increase in amygdala volume despite an overall cerebral reduction, suggests an abnormal brain development processes, particularly affecting medial brain regions (as amygdala and hippocampal formation), which is also in accordance with our previous evidence of absolute volumes preservation of hippocampal formation (Sampaio et al., 2010). Thus, limbic system volumes (amygdala and hippocampal formation) seem to be preserved in WS, when compared with typically developing individuals, and disproportionably enlarged when normalized to brain parenchyma. However, despite this evidence, fMRI studies show functional abnormalities in both regions (MeyerLindenberg, Hariri, et al., 2005; Meyer-Lindenberg, Mervis, et al., 2005). Moreover, if we take into account the neurodevelopment process, regions called proliferative zones are formed close to the ventricles which are associated with a rapid multiplying of neurons, and subsequently migrate to different targets in the cortex (Lenroot \& Giedd, 2006). Thus, one may hypothesize that genes deleted in the critical region of WS may play an important role in these neurodevelopment key events being associated with the preservation of the midline structures that are formed first during the neurodevelopmental process and display volume preservation. However, a disruption of signalizing and migration pathways to cortex may occur, as a consequence of gene deletion, explaining the absolute volume reduction of cortical gyri in WS (Reiss et al., 2004; Thompson et al., 2005). Of further note, in the WS critical deletion region, genes like FZD3 (Wang \& Bellugi, 1994) and FZD 9 (Zhao et al., 2005) are deleted; these are Wnt receptors that form part of the Wnt pathway, with important functions associated with the regulation of neural precursor proliferation during the early stages of development.

Furthermore, despite evidence of amygdala volume preservation, it is unclear how it would contribute to the hypersocial behavior of WS. Meyer-Lindenberg, Hariri, et al. (2005), Meyer-Lindenberg, Mervis, et al. (2005) found greater amygdala activation in response to threatening scenes than to threatening faces, as opposed to the pattern exhibited by typically developing controls (Meyer-Lindenberg, Hariri, et al., 2005). Considering the key role that the amygdala plays in monitoring danger, these authors proposed that reduced activation of the amygdala in individuals with WS, in response to threatening faces, might contribute to their reduced fear of strangers and consequent social disinhibition. A more recent fMRI study also report abnormal activation of the amygdala during face processing, thus providing additional evidence that the failure to recruit this area during face processing may be a neural correlate of the abnormally high sociability that characterizes this disorder (Paul et al., 2009). Consistent with these findings, other studies report abnormal patterns of activation in the amygdala (reduced activation to fearful faces and higher activation to happy faces) in WS (Haas et al., 2009), thus corroborating the link between low amygdala activation and higher approachability (Meyer-Lindenberg, Hariri, et al., 2005). Indirect evidence derived from neuropsychological studies also have proposed abnormal amygdala function in WS when performing emotion recognition tasks (Frigerio et al., 2006; Jones et al., 2000; Martens et al., 2009), despite others claiming that these abilities are also developmentally related (Capitao et al., 2011).

Future studies should focus on a detailed characterization of the social phenotype of individuals with WS, and functional MRI studies that address approachability and socio-affective ecological situations should be performed. Also, neuroimaging longitudinal pediatric studies should be conducted in individuals with WS in order to analyze the different processes in brain development that occur in this syndrome, as well as detailed developmental functional fMRI approaches.

\section{Acknowledgements}

This research was supported by the grants PIC/IC/83290/2007 from Fundação para a Ciência e Tecnologia (Portugal) and Fundación Alicia Koplowitz. (V convocatoria de 2009. Ayudas a la investigación en psiquiatría de la infancia y adolescencia enfermedades neurodegenerativas tempranas). We thank Nuno Sousa for his contributions for this research Project.

\section{References}

Adolphs, R. (1999). Social cognition and the human brain. Trends in Cognitive Sciences, 3(12), 469-479.

Adolphs, R. (2003). Cognitive neuroscience of human social behaviour. Nature Reviews in Neuroscience, 4(3), 165-178.

Adolphs, R., Tranel, D., Damasio, H., \& Damasio, A. (1994). Impaired recognition of emotion in facial expressions following bilateral damage to the human amygdala. Nature, 372(6507), 669-672.

Adolphs, R., Tranel, D., Damasio, H., \& Damasio, A. R. (1995). Fear and the human amygdala. Journal of Neuroscience, 15(9), $5879-5891$.

Altaye, M., Holland, S. K., Wilke, M., \& Gaser, C. (2008). Infant brain probability templates for MRI segmentation and normalization. Neuroimage, 43(4), 721-730.

Amaral, D. (2002). The primate amygdala and the neurobiology of social behavior: Implications for understanding social anxiety. Biological Psychiatry, 51(1), 1117.

Bellugi, U., Adolphs, R., Cassady, C., \& Chiles, M. (1999). Towards the neural basis for hypersociability in a genetic syndrome. Neuroreport, 10 (8), 1653.

Cahill, L., Babinsky, R., Markowitsch, H. J., \& McGaugh, J. L. (1995). The amygdala and emotional memory. Nature, 377(6547), 295-296.

Capitao, L., Sampaio, A., Fernandez, M., Sousa, N., Pinheiro, A., \& Goncalves, O. F. (2011). Williams syndrome hypersociability: A neuropsychological study of the amygdala and prefrontal cortex hypotheses. Research in Developmental Disabilities, 32(3), 1169-1179.

Chiang, M., Reiss, A., Lee, A., Bellugi, U., Galaburda, A., Korenberg, J., et al. (2007). 3D pattern of brain abnormalities in Williams syndrome visualized using tensorbased morphometry. Neuroimage, 36(4), 1096-1109.

Ewart, A., Morris, C., Atkinson, D., Jin, W., Sternes, K., Spallone, P., et al. (1993). Hemizygosity at the elastin locus in a developmental disorder, Williams syndrome. Nature Genetics, 5(1), 11-16.

Frigerio, E., Burt, D., Gagliardi, C., Cioffi, G., Martelli, S., Perrett, D., et al. (2006). Is everybody always my friend? Perception of approachability in Williams syndrome. Neuropsychologia, 44(2), 254-259. 
Galaburda, A., \& Bellugi, U. (2000). V. Multi-level analysis of cortical neuroanatomy in Williams syndrome. Journal of Cognitive Neuroscience, 12(Suppl. 1), 74-88. Galaburda, A., Rosen, G., \& Sherman, G. (1990). Individual variability in cortical organization: Its relationship to brain laterality and implications to function. Neuropsychologia, 28(6), 529-546.

Haas, B., Mills, D., Yam, A., Hoeft, F., Bellugi, U., \& Reiss, A. (2009). Genetic influences on sociability: Heightened amygdala reactivity and event-related responses to positive social stimuli in Williams syndrome. Journal of Neuroscience, 29(4), 1132.

Jernigan, T., Bellugi, U., Sowell, E., Doherty, S., \& Hesselink, J. (1993). Cerebral morphologic distinctions between Williams and Down syndromes. Archives of Neurology-Chicago, 50, 186-1186.

Jones, W., Bellugi, U., Lai, Z., Chiles, M., Reilly, J., Lincoln, A., et al. (2000). II. Hypersociability in Williams syndrome. Journal of Cognitive Neuroscience, 12(Suppl. 1), 30-46.

Klein-Tasman, B., \& Mervis, C. (2003). Distinctive personality characteristics of 8-, 9-, and 10-year-olds with Williams syndrome. Developmental Neuropsychology, 23(1), 269-290.

LeDoux, J. (1998). Fear and the brain: Where have we been, and where are we going? Biological Psychiatry, 44(12), 1229-1238.

Lenroot, R. K., \& Giedd, J. N. (2006). Brain development in children and adolescents: Insights from anatomical magnetic resonance imaging. Neuroscience and Biobehavioral Reviews, 30(6), 718-729.

Martens, M., Wilson, S., Dudgeon, P., \& Reutens, D. (2009). Approachability and the amygdala: Insights from Williams syndrome. Neuropsychologia, 47(12), 24462453.

Meyer-Lindenberg, A., Hariri, A., Munoz, K., Mervis, C., Mattay, V., Morris, C., et al. (2005). Neural correlates of genetically abnormal social cognition in Williams syndrome. Nature Neuroscience, 8(8), 991-993.

Meyer-Lindenberg, A., Kohn, P., Mervis, C., Kippenhan, J., Olsen, R., Morris, C., et al. (2004). Neural basis of genetically determined visuospatial construction deficit in Williams syndrome. Neuron, 43(5), 623-631.

Meyer-Lindenberg, A., Mervis, C. B., Sarpal, D., Koch, P., Steele, S., Kohn, P., et al. (2005). Functional, structural, and metabolic abnormalities of the hippocampal formation in Williams syndrome. Jounal of Clinical Investigation, 115(7), 1888-1895.

Paul, B., Snyder, A., Haist, F., Raichle, M., Bellugi, U., \& Stiles, J. (2009). Amygdala response to faces parallels social behavior in Williams syndrome. Social Cognitive and Affective Neuroscience, 4(3), 278-285.

Peoples, R., Franke, Y., Wang, Y., Pérez-Jurado, L., Paperna, T., Cisco, M., et al. (2000). A physical map, including a BAC/PAC clone contig, of the Williams-Beuren syndrome-deletion region at 7q11. 23. The American Journal of Human Genetics, 66(1), 47-68.

Pohl, K., Bouix, S., \& Kikinis, R. (2004). Anatomical guided segmentation with nonstationary tissue class distributions in an expectation-maximization framework. IEEE International Symposium on Biomedical Imaging, 81-84.

Reiss, A., Eckert, M., Rose, F., Karchemskiy, A., Kesler, S., Chang, M., et al. (2004). An experiment of nature: Brain anatomy parallels cognition and behavior in Williams syndrome. Journal of Neuroscience, 24(21), 5009.

Sampaio, A., Sousa, N., Fernandez, M., Vasconcelos, C., Shenton, M. E., \& Goncalves, O. F. (2010). Williams syndrome and memory: A neuroanatomic and cognitive approach. Journal of Autism and Developmental Disorders, 40(7), 870-877.

Scott, S. K., Young, A. W., Calder, A. J., Hellawell, D. J., Aggleton, J. P., \& Johnson, M. (1997). Impaired auditory recognition of fear and anger following bilateral amygdala lesions. Nature, 385(6613), 254-257.

Strømme, P., Bjømstad, P., \& Ramstad, K. (2002). Prevalence estimation of Williams syndrome. Journal of Child Neurology, 17(4), 269.

Tang, Y., Hojatkashani, C., Dinov, I. D., Sun, B., Fan, L., Lin, X., et al. (2010). The construction of a Chinese MRI brain atlas: A morphometric comparison study between Chinese and Caucasian cohorts. Neuroimage, 51(1), 33-41.

Thompson, P. M., Lee, A. D., Dutton, R. A., Geaga, J. A., Hayashi, K. M., Eckert, M. A., et al. (2005). Abnormal cortical complexity and thickness profiles mapped in Williams syndrome. Journal of Neuroscience, 25(16), 4146-4158.

von Gunten, A., Fox, N. C., Cipolotti, L., \& Ron, M. A. (2000). A volumetric study of hippocampus and amygdala in depressed patients with subjective memory problems. Journal of Neuropsychiatry and Clinical Neurosciences, 12(4), 493-498.

Wang, P., \& Bellugi, U. (1994). Evidence from two genetic syndromes for a dissociation between verbal and visual-spatial short-term memory. Journal of Clinical and Experimental Neuropsychology, 16(2), 317-322.

Zhao, C., Aviles, C., Abel, R. A., Almli, C. R., McQuillen, P., \& Pleasure, S. J. (2005). Hippocampal and visuospatial learning defects in mice with a deletion of frizzled 9 , a gene in the Williams syndrome deletion interval. Development, 132(12), 2917-2927. 